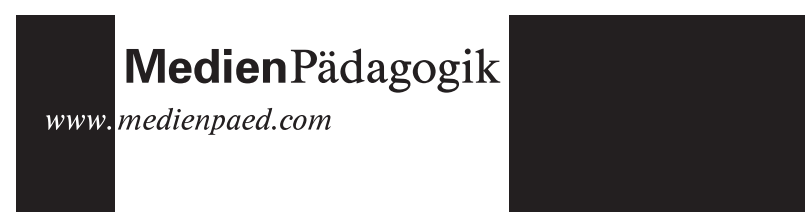

Anke Grotlüschen

24.5.2006

\section{Blended Learning für gering qualifizierte Lerner?}

Die seit langem bekannte〈Bildungsschere〉 weitet sich zu einer digitalen Diskriminierung aus: Einkommen und Vorbildung entscheiden darüber, wer an Blended Learning teilnehmen kann. Einige empirische Untersuchungen (v. a. das Berichtssystem Weiterbildung und die sog. MünchenStudie) zeigen auf, wer selbst gesteuert mit Medien lernt. Kontrastierend werden Evaluationsergebnisse eines Förderprojekts vorgestellt. Sie zeigen die Reichweite und Grenzen von E-Learning-Anteilen in Lernszenarien für gering qualifizierte Lernende auf.

E-Learning gilt als hochselektiv, denn bisher erreichen wir überwiegend Personen mit hoher Vorbildung, z. B. Führungskräfte mit ausgeprägten Fähigkeiten selbst gesteuert oder gar selbst bestimmt zu lernen. Dies gilt für alle Formen des Selbstlernens, wie das Berichtssystem Weiterbildung deutlich zeigt:

«In sozio-demographischen und regionalen Teilgruppen aller 19- bis 64Jährigen zeigen sich 2003 erhebliche Unterschiede im jeweiligen Anteil an Selbstlernern. An Selbstlernen (...) beteiligen sich 2003

- Beamte häufiger als Arbeiter (58\% vs. $26 \%$ );

- Personen mit einem Hochschulabschluss öfter als diejenigen ohne Berufsausbildung (53\% vs. 23\%);

- Personen mit Abitur häufiger als Personen mit niedriger Schulbildung (50\% vs. $25 \%$ );

- Deutsche öfter als Ausländer (36\% vs. $25 \%$ );

- 19- bis 34-Jährige häufiger als 50- bis 64-Jährige (39\% vs. $28 \%$ );
- Erwerbstätige öfter als Nichterwerbstätige (37\% vs. 30\%);

- Männer häufiger als Frauen (38\% vs. 32\%)»

(Berichtssystem Weiterbildung IX 2005, S. 60)

Es lässt sich weiterhin belegen, dass die Teilnahme an digital unterstütztem Lernen nach Milieus und Bildungshintergründen unterschiedlich ist (vgl. BSW IX 2005, Tippelt u. a. 2003). Diejenigen, die bereits über hohe Lernkompetenzen verfügen, bauen sie weiter aus, indem sie selbst gesteuert mit elektronischen Medien weiterlernen (s. u.). In diesem Aufsatz sollen die demografisch oder milieuspezifisch erhobenen Unterschiede kurz referiert werden. Darauf aufbauend kann dann die kleinere quantitative und vor allem qualitativ differenzierte Evaluationsstudie «Bildung als Brücke für Benachteiligte» eingeordnet werden.

\section{Doppelte Selektivität}

Im internationalen Diskurs wird das Phänomen der ungleichen Bildungsbeteiligung als kumulative Partizipation bezeichnet. Genauer wird der Prozess beschrieben als doppelte Selektivität (Faulstich 1981), mit der aufgezeigt wird, dass zunächst die Schule zwischen Gebildeten und weniger Gebildeten selektiert und anschliessend das Weiterbildungs- und Erwachsenenbildungssystem diese Selektion verschärft - anstatt sie zu kompensieren.

Ungleichheiten im Bildungssektor werden unter dem Schlagwort «Bildungsschere» zusammengefasst und sind an mehreren Studien abzulesen. Aktuell zeigt die Expertenkommission Finanzierung Lebenslangen Lernens, dass $13 \%$ der erwachsenen Bevölkerung nie an Weiterbildung teilnehmen (Expertenkommission 2004, S. 148 ff.). In der dazugehörigen Expertise ist genauer aufgeführt, dass diese Personen also dauerhaft nicht an Weiterbildung teilnehmen (Schröder, Schiel, Aust 2004, S. 84 ff.). Dies ist nur eine von mehreren Analysen, die darauf hinweisen, dass sich Weiterbildung in Form von einer Schere verhält. Weitere Umfragen zur Weiterbildung, zum Beispiel das Berichtsystem Weiterbildung, das sozioökonomische Panel, die Erhebungen des Bundesinstitutes für Berufsbildung und des IAB sowie der Mikrozensus (Feller 2004, S. 120) stützen dieses Ergebnis. Des Weiteren zeigen diese Teilnehmerbefragungen, dass zum ersten Mal seit 1979 die Teilnahme an Weiterbildung rückläufig ist (Berichtssystem Weiterbildung VIII \& IX). Genau genommen folgt Weiterbildung noch immer der «More-and-more-Regel» (Michel, Pelka 2003, S. 
19). Das besagt, dass Weiterbildung vor allen Dingen von den Personen in Anspruch genommen wird, die ohnehin schon über hohe formale Bildung verfügen. Dieser Trend zeigt sich seit 1979 und ist auch 2003 wieder erkennbar. So nehmen beispielsweise von den Personen mit niedriger Schulbildung etwa $28 \%$ an Weiterbildung teil, während von den Personen mit Abitur 59\% in Weiterbildungsangeboten zu finden sind (BSW IX, vgl. Abb. 1). Dieses Prinzip der selbst verstärkenden Effekte wird als MatthäusPrinzip bezeichnet (vgl. Ehmann 2003, Tippelt u. a. 2003).

\section{Tabelle 6.5: Teilnahme an Weiterbildung nach beruflicher Qualifikation 1979 - 2003}

Teilnahmequoten in \%

Abbildung 1: Berichtssystem Weiterbildung IX, S. 29

\begin{tabular}{|c|c|c|c|c|c|c|c|c|c|}
\hline Weiterbildiung insgesamt & & & & & & & & & \\
\hline Keine Berufsausbildung & 12 & 11 & 9 & 18 & 18 & 19 & & & \\
\hline $\begin{array}{l}\text { Lehre / Berufsfachschule } \\
\text { Meister. }\end{array}$ & 21 & 27 & 22 & 32 & 33 & 39 & 45 & 40 & \\
\hline $\begin{array}{l}\text { Meister-, andere Fachschule } \\
\text { Hochschuabschluss }\end{array}$ & 32 & 36 & 39 & 49 & 48 & 52 & 58 & 54 & 55 \\
\hline Hochschulabschluss & & & & 53 & 59 & 64 & 69 & 63 & 62 \\
\hline Allgemeine Weiterbilidung & & & & & & & & & \\
\hline Keine Berufsausbildung & 10 & & & & 13 & & 19 & & \\
\hline Lehre / Berufsfachschule & 16 & 20 & 15 & 20 & 20 & 24 & 29 & 24 & 23 \\
\hline $\begin{array}{l}\text { Meister-, andere Fachschule } \\
\text { Hochschulabschluss }\end{array}$ & $\begin{array}{l}20 \\
31\end{array}$ & ${ }_{38}^{22}$ & $\begin{array}{l}26 \\
32\end{array}$ & ${ }_{34}^{27}$ & $\begin{array}{l}25 \\
36\end{array}$ & $\begin{array}{l}27 \\
40\end{array}$ & $\begin{array}{l}34 \\
45\end{array}$ & $\begin{array}{l}31 \\
41\end{array}$ & $\begin{array}{l}32 \\
38\end{array}$ \\
\hline Berufliche Weiterbildung & & & & & & & & & \\
\hline 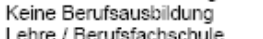 & & 2 & 12 & 5 & 78 & 5 & 29 & ${ }_{27}^{9}$ & \\
\hline $\begin{array}{l}\text { Lehre / Berusfachschule } \\
\text { Meister-, andere Fachschule }\end{array}$ & 20 & 19 & 24 & 32 & 34 & 36 & 42 & ${ }_{42}^{27}$ & $\begin{array}{l}24 \\
38\end{array}$ \\
\hline Hochschulabschluss & 24 & 36 & 27 & 34 & 39 & 43 & 48 & 43 & 44 \\
\hline
\end{tabular}

Abbildung 2: Berichtssystem Weiterbildung IX, S. 29

Dabei rückt mehr und mehr das Problem des sozialen Zusammenhalts in den Mittelpunkt. Zu befürchten ist eine Verfestigung der Teilnahmequoten nach Milieus bzw. Schichten. So sind laut der PISA-Erhebung 2000 Deutschland und die Schweiz die Länder, in denen die Lesekompetenz von Jugendlichen aus höheren und niedrigen Sozialschichten die grössten Unterschiede aufweist (Baumert u. a. 2001, S. 383). Auch die Weiterbildung verzeichnet seit den allerersten Untersuchungen eine relativ dauerhafte Selektivität. Allerdings kommt die ehemalige Benachteiligungsformel nicht mehr ganz zum Tragen, da Religion und Stadt/Land-Unterschiede nicht mehr so relevant sind (Vester 2004). Die «katholische Arbeitertochter vom Lande», welche Religion, Herkunftsfamilie, Geschlecht und Region als klassische Benachteiligungsfaktoren benennt, reduziert sich heute auf die «Arbeitertochter». Dies hat auch mit erheblichen Vorurteilen gegenüber der Herkunftsfamilie zu tun, wie sich an der Überprüfung der Gymnasialempfehlungen/Lernausgangslagen für Kinder aus unterschiedlichen Sozialschichten zeigt: Ein Kind aus schwächerer Sozialschicht braucht mindestens 90 Leistungspunkte für eine Gymnasialempfehlung, während ein Kind aus besserem Hause diese bereits mit 65 Punkten bekommt (Lehmann, Peek, Gänsfuss 1997).

\section{Digitale Beteiligung - Digitale Diskriminierung}

Dasselbe Phänomen findet sich hinsichtlich digitaler Beteiligung: Erstens ist der Besitz digitaler Medien und Zugänge nach Bildungsstand und Einkommen unterschiedlich und zweitens ist die Nutzung digitaler Lernszenarien von diesen Faktoren abhängig: es entsteht eine digitale Diskriminierung. Die Bildungsschere wiederholt und verschärft sich bei der digitalen Beteiligung der Bevölkerung. Hier gibt vor allen Dingen der sogenannte (N)ONLINER-Atlas Auskunft, der von der TNS Emnid und der Initiative D21 in Auftrag gegeben wird. Aus dem Atlas 2004 geht hervor, dass die $41 \%$ der Deutschen Offliner «mehrheitlich weiblichen Geschlechts sind, formal eine geringe Bildung und ein niedriges Einkommen haben».

Zudem weist das sozioökonomische Panel bereits seit 2000 daraufhin, dass Einkommen und Bildung darüber entscheiden, ob jemand das Internet nutzt oder nicht (DIW Wochenbericht 41/00). Es ist in aller Deutlichkeit festzuhalten, dass die Technologienutzung als zusätzliche Hemmschwelle für alle diejenigen wirkt, die über Technologie aus materiellen Gründen nicht verfügen oder die über die Nutzungskompetenzen nicht verfügen. Diese Feststellung zeichnet aus pädagogischer Sicht ein kritisches Bild für E-Learning. Eine bewahrpädagogische Verhinderungsstrategie kann jedoch nicht die Lösung sein. Viel eher ist eine Strategie sinnvoll, die die bildungs- und technologiebenachteiligten Gruppierungen einbezieht. Dazu ist es notwendig, die besonderen Kennzeichen digitalen Lernens zu kennen und hier gezielte Unterstützungsvarianten zu entwickeln.

\section{Weiterbildungsbarrieren - E-Learning-Barrieren}

Kommen wir noch einmal auf die unterschiedliche Nutzung von Weiterbildung sowie die digitale Spaltung zurück. Es gibt hierzu aktuelle Untersuchungen, die herausstellen, welche Art von Barrieren einen Teil der Bevölkerung davon abhalten, regelmässig und intensiv an Weiterbildung 
teilzunehmen (Kuwan 2004, Tippelt u. a. 2004). Ich greife diese Barrieren kurz auf um zu prüfen, inwieweit sie auch für die Nutzung von E-Learning sowie für eine digitale Beteiligung zutreffen.

Erstens handelt es sich um eine fehlende Nutzenerwartung des Lernens. Lernen wird besonders von benachteiligten (und oft arbeitssuchenden) Lernern ausschliesslich als Instrument zur Erhaltung der Arbeitsfähigkeit betrachtet. Wenn kein solcher Nutzen erkennbar ist, wird an Weiterbildung auch nicht teilgenommen. Wir kennen diese fehlende Nutzenerwartung auch hinsichtlich E-Learning schon länger unter dem immer wieder verwendeten Begriff «Mehrwert von E-Learning». Dahinter verbirgt sich die Frage, ob denn ein wirklicher Mehrwert des E-Learning gegenüber anderen Lehr-Lernformen zu erkennen ist. Diese Frage ist meines Erachtens nach wie vor offen

Zwei weitere Weiterbildungsbarrieren sind das Alter sowie die Ablehnung formal organisierter Weiterbildung. Die digitale Beteiligung ist altersspezifisch verteilt, insofern wirkt die Digitalisierung der Weiterbildung eher noch verschärfend auf die Verteilungsschere. Hinsichtlich der Ablehnung formal organisierter Weiterbildung kann E-Learning durchaus entlastend wirken.

Die vierte Weiterbildungsbarriere besteht darin, dass die Befragten keinen Spass am Lernen haben bzw. negative Lernerfahrungen anführen. Hier ist zu vermuten, dass E-Learning durch den Neuigkeitseffekt erst einmal etwas unabhängiger von den bisherigen Lernerfahrungen wahrgenommen wird.

Schlechte Lernerfahrung geht einher mit Angst vor Misserfolg und einem erhöhten Beratungs- und Unterstützungsbedarf. Diese zwei Elemente werden durch die Digitalisierung eher verstärkt, da sich das Frustrationspotenzial des Allein-Scheiterns vor dem PC sowie das Phänomen «Lost in Hyperspace» zu den ohnehin vorhandenen Verlassenheitsgefühlen hinzugesellen (alle referierten Barrieren: Kuwan 2004, S. 178). Ganz ähnliche Barrieren werden einschliesslich einer Priorisierung und Milieuzuordnung in der so genannten München-Studie vorgebracht (Tippelt u. a. 2004, S. 293).

\section{Lernprozesse gering qualifizierter Personen}

Es gibt insofern Gründe für bestimmte Bevölkerungsgruppen, nicht $\mathrm{zu}$ lernen. Der überwiegende Teil dieser Gründe wird durch E-Learning nicht abgebaut, sondern verfestigt. Doch was geschieht, wenn Angehörige eben dieser Gruppierungen dennoch lernen und dazu aufgefordert sind, sich mit
Lernsoftware selbst gesteuert an eine Fülle möglicher Teilthemen anzunähern? Wir wissen wenig über diese Lernprozesse. Durch Beobachtungen und Befragungen konnten aus einem Hamburger Projekt Einblicke bezogen werden. Zielsetzung des ehrgeizigen Projekts war unter anderem, die Beteiligung weniger qualifizierter Lerner an E-Learning zu erhöhen. Das Projekt setzt sich zusammen aus einem Konsortium, in dem Bildungsträger, Beschäftigungsträger und Software-Entwickler beteiligt sind. Gefördert wurde das Vorhaben mit dem Titel «Information and Communication Competence - Bridge to the Market» von der Freien und Hansestadt Hamburg, vom Bundesministerium von Wirtschaft und Arbeit sowie von der EU-Gemeinschaftsinitiative EQUAL. Evaluiert wurde das Projekt durch die Universität Hamburg (wir beziehen uns hier auf die erste Erhebungsrunde und ihre Ergebnisse). Ziele des Projekts waren unter anderem:

1. Tele-Lernen für gering qualifizierte Personen anzubieten,

2. zur Überwindung der digitalen Spaltung beizutragen sowie

3. zur Erhöhung von Selbstlernkompetenzen dieser Zielgruppe beizutragen.

Zur Durchführung werden Kurse bereitgestellt, bei denen zunächst Computer-Software erlernt wird (z. B. Tabellenkalkulations-Software, Textverarbeitungs-Software, Internet-Browser und Mail-Software). Diese Kurse wurden jedoch nicht in klassischer Form bereitgestellt, sondern mit Hilfe einer Lern-Software zunächst im Schulungsraum unter fachkundiger Anleitung von Lehrkräften ${ }^{1}$ abgehalten. In einer zweiten Projektphase wird nach der Informationskompetenz, die durch die Kenntnis von StandardSoftware verbessert werden soll, zunehmend Kommunikationskompetenz trainiert. Hierzu wird eine eigens entwickelte Software eingesetzt, in der es im Wesentlichen um Kundenorientierung und Kommunikation geht. Dabei wird angenommen, dass für die angesprochene Zielgruppe im Dienstleistungssektor auf dem Arbeitsmarkt Beschäftigungsmöglichkeiten vorhanden sind, für die sie qualifiziert werden sollen. Die Erhebungsrunde zum Themenfeld Kundenorientierung läuft weiterhin, erste Ergebnisse liegen vor, sind jedoch noch nicht publiziert.

\footnotetext{
Die Lehrkräfte befanden sich selbst in ihrer eigenen Weiterbildung zum «Experten neuer Lerntechnologien» (Teleakademie Furtwangen).
} 
Aus mehreren hundert Teilnehmenden des Projekts wurde die Erhebung zugespitzt auf 45 Personen, die direkt in den ECDL-Kursen ${ }^{2}$ erreichbar waren. Diese Personen wurden durch Vorher- und Nachher- Fragebögen befragt, mit Hilfe von strukturierten Beobachtungen wurden in der Erhebung sieben intensivere Fallanalysen gewonnen. Die Beobachtungen fanden während circa Zweidrittel der Kurstage statt, dabei waren jeweils einer oder zwei Beobachterinnen durchgehend im Kursraum anwesend. Als Erhebungsinstrumente wurden erstens ein Eingangsfragebogen eingesetzt, bei dem es sich um eine syllabusgebundene Selbsteinschätzung handelt. Zum Kursende folgte der Ausgangsfragebogen, so dass eine Vorher-Nachher-Analyse möglich wurde. Die Fragebögen wurden auf Plausibilität geprüft und mit den Ergebnissen der Beobachtung abgeglichen. Die Beobachtung selbst basierte auf lerntheoretischen Kategorien. (Grotlüschen/ Brauchle 2004, S. 165 und S. 57) Zusätzlich zu den Beobachtungen wurden Beobachtungstranskripte angefertigt, in denen Originalsequenzen von Dialogen und Handlungen der speziell beobachteten Teilnehmenden direkt niedergeschrieben wurden.

\section{Lerntheoretischer Hintergrund}

Zur Entwicklung der Fragebögen sowie des Beobachtungsbogens diente uns die subjektwissenschaftliche Lerntheorie (Holzkamp 1993). Der subjektwissenschaftliche Ansatz geht von intentional handelnden Personen aus (a. a. O. S. 23), welche für ihre Handlungen jeweils eigene Gründe haben. Aus der Perspektive des Subjekts ist somit die Handlung vernünftig, auch wenn sie aus dritter Perspektive irrational und unverständlich erscheint. Dieses Paradigma wird als «Begründungsdiskurs» bezeichnet (a. a. O. S. 27ff). Die qualitativen Teile der referierten Erhebung bedienen sich daher einer Perspektive, die die subjektiven Gründe der Handelnden zu verstehen versucht. Zwei Einschränkungen sind dabei zentral: erstens gehen wir davon aus, dass wir den Gegenüber nicht in seiner Vollständigkeit ergründen können, zumal uns nur kurze Ausschnitte aus dem Leben der Beobachteten und Befragten zugänglich sind. Zweitens sind subjektive Gründe keineswegs als utilitaristische Nutzenoptimierung zu verstehen, sondern sie enthalten Unzulänglichkeiten, Emotionen, Widersprüche und allerhand Unerwartetes, das sich von kühlem Abwägen verschiedener

\footnotetext{
2 European Computer Driving Licence, ein europaweit einheitliches Zertifikat über die Kenntnis von Standardsoftware. Bei vier von sieben möglichen Prüfungen wird der ECDL START verliehen.

Alternativen unterscheidet (a. a. O., S. 24, vgl. auch Holzkamp 1997, S. 255ff).

Bezogen auf Lernen als spezifische Handlungsform liefert Holzkamp eine differenzierte Begrifflichkeit, die den empirischen Zugriff erleichtert. Wir beziehen uns hier speziell auf Teilprozesse, die am Anfang einer Lernhandlung stehen sowie Prozesse, die die Interaktion zwischen Lehrenden, Gruppe und Lernenden betreffen. Dabei blenden wir Prozesse des Verlaufs sowie institutionelle Rahmungen - die in der subjektwissenschaftlichen Lerntheorie differenziert zueinander in Bezug gesetzt werden - in Teilen aus. Holzkamp greift hinsichtlich gesellschaftlicher Analysen auf das Werk «Überwachen und Strafen» zurück, das Michel Foucault vorgelegt hat (1977). Für unseren Ansatz ist jedoch das aktuell diskutierte Modell der Milieuforschung relevanter, da wir so zu genaueren Informationen über die hier betroffenen Zielgruppen erhalten. Der zugrunde liegende Ansatz von Bourdieu wird im Anschluss an die lerntheoretischen Begriffe kurz dargelegt.

Lerntheoretisch haben wir auf den Kernbegriff der Diskrepanzerfahrung (a. a. O. S. 211ff) zurückgegriffen. Es handelt sich dabei um die Erfahrung, dass die aktuellen Handlungsanforderungen nicht mit gegebenem Repertoire vollzogen werden können. Zwischen den Anforderungen und den Möglichkeiten entsteht die besagte Diskrepanz. Um aus diesem Zustand zum Lernen überzugehen, muss jedoch eine zweiter Faktor erfüllt sein: der oder die Lernende muss davon ausgehen, dass er/sie mit Hilfe des Lernens das eigentliche Problem erfolgreich bewältigen kann. Dies Phänomen diskutiert Holzkamp als Antizipation lernender Überwindung des Problems (a. a. O. S. 190f).

Als drittes Element ist die Interaktion zwischen Gruppe und Lehrkraft wichtig (a. a. O., S. 385ff), die wir ebenfalls im Rahmen des strukturierten Beobachtungsbogens betrachtet haben. Aus diesen Kernelementen subjektwissenschaftlicher Lerntheorie speisen sich sowohl Eingangs- als auch Ausgangsbefragung und der Beobachtungsbogen. Die Fallanalysen nehmen diese jeweiligen Elemente wieder auf. Der Beobachtungsbogen, die Fragebögen, die vollständigen Fallanalysen - systematisiert nach Diskrepanzerfahrung, Antizipation und Interaktion - würden hier den Rahmen sprengen. Sie sind an anderer Stelle vollständig abgebildet (Grotlüschen/ Brauchle 2004).

Für diesen Beitrag sollen einzelne, über die theoretischen Kategorien hinaus in den Fallanalysen aufgetauchte Hinweise hervorgehoben werden. 
Dies soll dazu dienen, Eigenheiten benachteiligter Lernender aufzuzeigen. Die Frage, ob Benachteiligte überhaupt von E-Learning profitieren, soll hier lediglich mit einer dichten Beschreibung auf qualitativer Basis und mit vorsichtigen Hinweisen aus der quantitativen Empirie bearbeitet werden. Die geringen Fallzahlen des quantitativen Erhebungsteils lassen keine weitere Verallgemeinerung $\mathrm{zu}$, sondern informieren als Vollerhebung der Kurse über deren Erfolg.

\section{Sozial unten stehende Milieus, Weiterbildung und E-Learning}

Der zweite theoretische Rückgriff bezieht sich auf das Habituskonzept nach Pierre Bourdieu (1987). Gegenüber der klassischen demografischen Forschung schlägt die milieudifferenzierte Forschung eine zusätzliche, horizontale Differenzierung vor, mit der gesellschaftliche Gruppen gezielter beschrieben werden (Überblick: Bremer in Vorb.). Dies Konzept hat weite Verbreitung mit dem Begriff Milieus gefunden. Die auf qualitativer Basis entwickelten Milieus werden in Deutschland für verschiedene Forschungsfragen verwendet. Zwei aktuelle Erhebungen beziehen sich auf Weiterbildung (Tippelt, Weiland, Panyr, Barz 2003; Barz, Tippelt 2004). Die erste Erhebung ist hinsichtlich der Vorgehensweise transparenter publiziert, daher beziehen wir uns im Wesentlichen auf die 2003 veröffentlichte so genannte München-Studie. Einige Ergebnisse werden hier kurz referiert, um Hinweise auf Weiterbildungsverhalten sozial unten stehender Milieus zu erhalten. Wir haben die München-Studie und ihre Ergebnisse herangezogen, um zu sehen, ob sich dadurch weitere Aspekte der Fallanalysen aufklären lassen.

Betrachten wir die hier relevante Zielgruppe gering qualifizierter Lernender: Es handelt sich durchweg um Personen in Arbeitsbeschaffungsmassnahmen, also um Beschäftigte am zweiten Arbeitsmarkt. Wir können davon ausgehen, dass in dieser Gruppierung ein geringes Einkommen vorhanden ist. Wir wissen, dass es sehr unterschiedliche Herkunftsländer gibt, zum Teil auch Schwierigkeiten mit der deutschen Sprache. Wir wissen jedoch relativ wenig über die Einstellung gegenüber Bildung und Weiterbildung. Bezieht man diese Gruppierung auf die Milieu-Grafiken der MünchenStudie, kommen aufgrund des geringen Einkommens drei Milieus in Frage, in denen wir unsere gering qualifizierten Lernenden antreffen könnten. Das sind erstens die so genannten Traditionsverwurzelten, eine Kriegsgeneration, die sich weitgehend dadurch auszeichnet, dass sie heute in die Jahre gekommen ist, überwiegend nicht mehr arbeitet und auch an Weiter- bildung wenig teilnimmt, weil der Bildung ohne Berufsrelevanz wenig Bedeutung beigemessen wird. Ein zweites Milieu, das im unteren materiellen Bereich angesiedelt ist, sind die Konsum-Materialisten. Hier wird die mangelnde wirtschaftliche Ausstattung durch deutliche Konsumorientierung kompensiert - und zwar trotz der mangelnden finanziellen Möglichkeiten. Einige Aktivitäten der beobachteten Gruppe liessen sich durchaus dem Feld der Konsum-Materialisten zuordnen. Eine dritte Gruppe sind die Hedonisten, ebenfalls im unteren materiellen Bereich, jedoch im postmodernen Einstellungsbereich anzusiedeln. Hier finden sich Haltungen der Verweigerung auf Basis der Zugehörigkeit zur herrschenden Gesellschaft. Das heisst, es kommt zu Provokationen und Abweichungen, jedoch immer auf Basis des Grundgefühls, dass Hedonisten sich als Teil der Gesellschaft empfinden, gegen die sie sich auflehnen ${ }^{3}$. Diese drei Gruppen verhalten sich unterschiedlich zu Weiterbildung. Für neuen Formen des Lernens, wie z. B. E-Learning, liegt eine differenzierte Auswertung der für München repräsentativen Erhebung vor (vgl. Abb. 2).

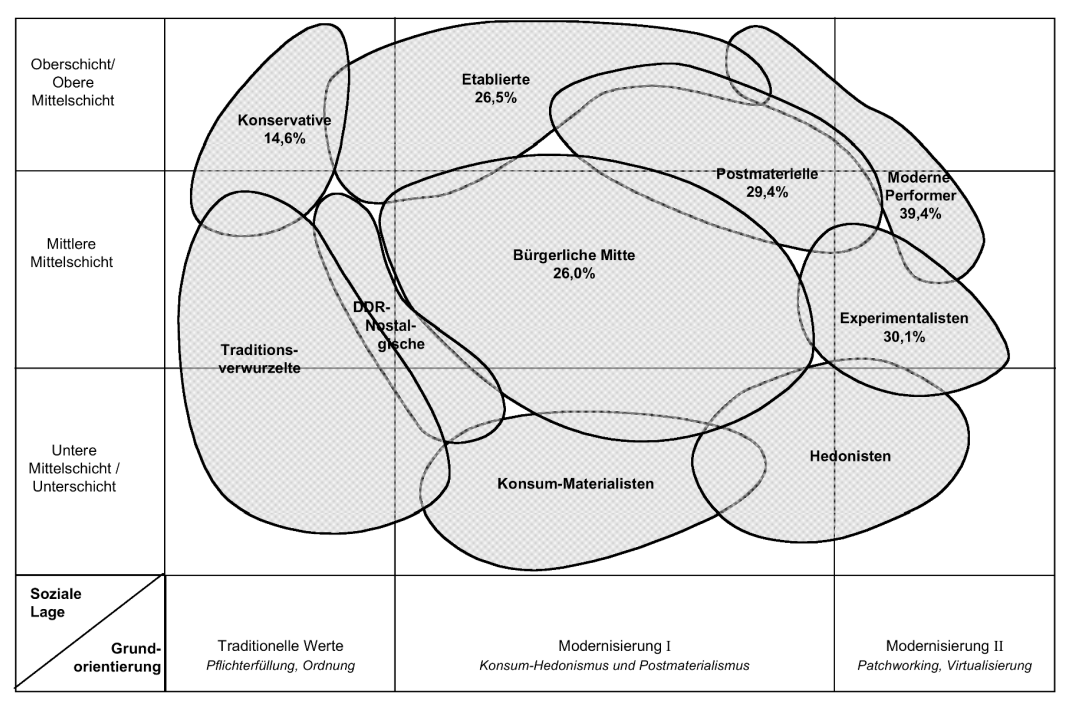

Abbildung 3: Selbst gesteuertes Lernen mit Medien, aus: Tippelt u. a. 2003, S. 155

${ }^{3}$ Was hinsichtlich der Milieustudien vollkommen offen bleibt, ist die Frage von Migrantinnen und Migranten. Hier ist immer kritisiert worden und lässt sich auch durch die München-Studie nicht weiter lösen. 
Die Abbildung 2 «Selbstgesteuertes Lernen mit Hilfe von Medien» weist auf zwei Dinge hin. Erstens erkennt man eine durchschnittliche Quote von 28,1\% der Münchener Befragten, die von sich angeben, sie nähmen an Lernprozessen teil, die sie selbst steuerten und $\mathrm{zu}$ denen sie Medien einsetzten. Zweitens sieht man anhand der milieuzugeordneten Quoten, dass im rechten Bereich der modernen Performer (39,4\%), der Experimentalisten $(30,1 \%)$ sowie der Postmateriellen (29,4\%) eine erheblich höhere Neigung zum selbst gesteuerten Lernen mit Hilfe von Medien zu verzeichnen ist. Weiterhin zeigt sich in konservativeren und etablierten Bereichen sowie in der Mitte eine eher schwächere Beteiligung.

Was aber deutlich auffällt, sind fehlende Daten der Traditionsverwurzelten, der Konsum-Materialisten und der Hedonisten, das bedeutet: Diese Milieus entziehen sich den Befragungen. Insofern haben wir keinen Einblick in die Präferenzen hinsichtlich selbst gesteuerten Lernens mit Medien. Die nachfolgende bundesweite Studie «Weiterbildung und soziale Milieus in Deutschland» (Barz, Tippelt 2004) hat diese Frage nicht mehr explizit ausgewiesen, sodass wir auf Bundesebene wiederum keine Daten zum Themenfeld haben. Unsere eigene Erhebung erlaubt keine Verallgemeinerung der Daten, jedoch können wir annehmen, exakt diese befragungsresistenten Gruppen durch die Erhebungsinstrumente erreicht zu haben. Dies konnte nur durch die Befragung innerhalb von Kursen gelingen. In einer nachfolgenden Erhebung haben wird diese Befragungen variiert und fortgesetzt, so dass zunehmend dichtere Daten über Benachteiligte hinsichtlich ihrer Lernhandlungen mit Neuen Medien gewonnen werden (Grotlüschen/ Brauchle im Druck)

\section{Exemplarische empirische Ergebnisse}

Wir können mit der Evaluation des Projekts Information and Communication Technology Competence - Bridge to the Market zwar nur in geringem Umfang quantitative Daten liefern, jedoch können aufgrund der Nähe zu den Beteiligten und der direkten Beobachtung einige qualitative Eindrücke liefern, die darauf schliessen lassen, wie hoch die Bereitschaft ist, sich auf selbst gesteuertes Lernen mit Hilfe von Medien einzulassen. Um einige Ergebnisse exemplarisch vorzustellen, greife ich auf den Kurs Tabellenkalkulation (Excel) zu. Es handelt sich hier um einen zweiwöchiges Seminar, an dem 15 Personen teilgenommen haben. Etwa die Hälfte der Unterrichtszeit wurde mit einer Lern-Software zugebracht, die multimedial durch audiovisuelle Elemente die Inhalte präsentiert. Sie stellt weiterhin
Übungen und Testelemente bereit. Ziel der Teilnahme am Excel-Kurs ist das Bestehen der ECDL-Prüfung. Hier ergibt sich eine Besonderheit der Erhebung: Da es ein europaweit einheitliches Curriculum gibt - den ECDL-Syllabus - haben wir einen grossen Teil der Fragebogenerhebung darauf ausgerichtet (s. u.).

\section{Frau Unsichtbar, Herr Selfmade und Frau Bockig}

Einige Fallanalysen lassen erkennen, dass die Herangehensweise gering qualifizierter Lerner an E-Learning durchaus unterschiedlich ist. Wir haben zur besseren Nachvollziehbarkeit alle fallanalytisch beobachteten Personen mit Fantasienamen belegt. Diese Namen charakterisieren den einen oder andern typischen Lernzug der Personen und spitzen die Fallanalyse in gewisser Weise zu.

Die erste Person über die wir hier berichten wollen, haben wir benannt als Frau Unsichtbar. Frau Unsichtbar bezeichnet sich selbst als langsam, sagt aber weiterhin von sich, dass ihr Fleiss sich auszahle. Es kann also davon ausgegangen werden, dass sie eine durchaus willige Lernerin ist, die durchaus eine Überwindung ihrer Handlungsprobleme durch Lernen antizipiert. Sie ist bereit, auch abends nach dem eigentlichen Kurs zu lernen oder sich von ihrem Sohn weitere Elemente zeigen zu lassen. Wie sich im Verlauf des Kurses herausstellt, ist diese Technik des langsamen und konsequenten Vorgehens für Frau Unsichtbar durchaus adäquat. Trotzdem schafft es Frau Unsichtbar nicht, eine der vier angebotenen ECDL-Prüfungen erfolgreich abzulegen. Frau Unsichtbar hat insgesamt 44 GrundlagenKapitel und 6 Excel-Kapitel bearbeitet. Man kann also davon ausgehen, dass sie einen gewissen Teil des Unterrichtsstoffes eigenständig erworben hat.

Im Verhältnis zwischen Präsenz und E-Learning zeigt sich für Frau Unsichtbar durch das E-Learning eine deutliche Erleichterung. Diese besteht darin, dass sie sich ihr Lerntempo eigenständig auswählen kann. Das ist jedoch nicht der entscheidende Faktor. Der weitaus anstrengendere Teil ist für sie die synchron durchgeführte Gruppenübung. Diese löst bei ihr Nervosität und Überforderung aus. Sie geht praktisch im Gruppengeschehen unter und äussert das durch Gesten und Aussagen, mit denen sie ihre Hilflosigkeit gegenüber ihren Nachbarn und Nachbarinnen und der Beobachterin zu erkennen gibt. Hinsichtlich der Interaktion in der Gruppe profitiert sie somit von der Individualisierung durch E-Learning. Die ELearning-Phasen, in der sie aufgefordert ist, die Übungen für sich allein 
durchzugehen, geben ihr Zeit zur Erholung, um in Ruhe zu arbeiten, ohne sich vor der Gruppe oder der Lehrkraft zu blamieren. Anhand der durchgegangenen Kapitel können wir weiterhin feststellen, dass Frau Unsichtbar als E-Learning-Anfängerin dazu neigt, die Kapitel von vorne nach hinten in der vorgegebenen Reihenfolge durchzuarbeiten. Diese Herangehensweise ist nicht sehr effizient und müsste bei gering qualifizierten Lernern sowie bei allen anderen Anfängern im E-Learning thematisiert und gegebenenfalls durchbrochen werden.

Auf der anderen Seite der Temposkala befindet sich im selben Kurs ein Mann namens Herr Selfmade. Herr Selfmade gibt sich sehr bildungsbeflissen und aufstiegsorientiert und grenzt sich von anderen Mitgliedern der Gruppe ab. Dies ist für die drei benannten Milieus in keiner Weise typisch. Die Unterschichtmilieus neigen eher dazu, sich solidarisch zueinander zu verhalten. Insofern irritiert Herr Selfmade durch seine abweisende Herangehensweise, z. B. durch seine Kritik an Meinungen und Erfahrungen anderer Kursmitglieder. Herrn Selfmade lernt relativ schnell, er schreitet in der Software zügig voran, lernt in den Pausen weiter, lernt auch anstelle einer Betriebsversammlung und berichtet mit Stolz von seinen bisher glänzenden Prüfungsergebnissen. Er hat alle vier Prüfungen für den ECDLStart abgelegt und hat sich zum Ziel gesetzt, den vollständigen ECDL mit allen sieben Prüfungen zu bestehen. Er fragt unter anderem danach, ob die Universität Hamburg ihm den dazu nötigen Kurs zur Software 〈Access〉 bereitstellen kann. Eine präzise Diskrepanzerfahrung bleibt empirisch zwar unklar - wir wissen nicht, wozu Herr Selfmade den ECDL speziell einsetzen möchte - dennoch ist die Antizipation, durch Lernen seine gegenwärtigen Handlungsbeschränkungen überwinden zu können, offenbar gegeben. Die Interaktion mit der Gruppe löst auch Herr Selfmade durch den Rückzug in individuelles Lernen innerhalb des Kurssettings.

Es ist also zunächst einmal erkennbar, dass sowohl Frau Unsichtbar als auch Herr Selfmade im selben Kurs mit Hilfe der Software ihrem eigenen Tempo entsprechend lernen können, mit anderen Worten: E-Learning ist für Binnendifferenzierung innerhalb von Kursen durchaus geeignet. Einen wichtigen weiteren Hinweis liefert zudem die Milieutheorie hinsichtlich des Habitus des Herrn Selfmade. Selfmade äussert seine Ansicht, jeder sei seines eigenen Glückes Schmied, mit den Worten: «entscheidend ist, ob man es will» und erklärt, er wolle die Sachen lernen - «und dann geht es auch!» Er distinguiert sich mit diesen Einstellungen von anderen Teilnehmern der Gruppe. Dies zeigt sich vor allem daran, dass er den frustrier- ten Teilnehmern Nr. 18, 19 und 20 mit Gesten der Überlegenheit erklärt: «Erfolg mit Lern-Software hängt von den Fähigkeiten von jedem [Einzelnen] ab!». Diese Distinktion lässt sich nur erklären, wenn man davon ausgeht, dass der Habitus des Herr Selfmade durch die Unterschichtmilieus falsch verortet ist und eher durch eines der Mittelschichtmilieus oder den oberen Bereich der Traditionsverwurzelten erklärbar ist. Dies ist durchaus möglich und würde auch mit seiner Grundeinstellung korrespondieren, der zufolge Bildung an sich sinnvoll und wichtig sei und nach Möglichkeit intensiv genutzt werden müsse (Diese Einstellung zeigt sich z. B. am Weiterlernen während der Pausen).

Auch seine Aufstiegsorientierung weist darauf hin, dass sein Habitus eher innerhalb der mittleren Mittelschicht der Traditionsverwurzelten oder in der bürgerlichen Mitte verortet sehen müsste.

Eine dritte Fallanalyse, auf die ich kurz zurückgreifen möchte, nennen wir «Frau Bockig». Frau Bockig erweist sich aus unserer Sicht als äusserst widerständig gegenüber der Lern-Software. Sie ist nicht gewillt, sich mit der Software dem Thema Tabellenkalkulation anzunähern. Sie geht zudem davon aus, dass ihr Mathematik nicht besonders läge, dass sie nicht rechnen könne und diese Erfahrung bereits in der Schule gemacht habe. Sie geht davon aus, dass die eigenständige Aneignung der Inhalte mit einer Software eher schwieriger für sie sei als die Erklärung durch das Lehrpersonal. Sie vermutet deshalb, durch die E-Learning-Anteile in einem Tabellenkalkulationskurs überfordert zu sein.

Mit dieser Selbsteinschätzung irritiert sie sowohl die Beobachterinnen als auch die Lehrkraft. Letztendlich stellt sich heraus, dass Frau Bockig mathematisch sehr wohl im Stande ist, die notwendigen Übungen zu berechnen und inhaltlich zu verstehen und dass sie weiterhin ganz hervorragend mit dem Tabellenkalkulationsprogramm umgeht. Mit anderen Worten: Ihre Widerständigkeit muss andere Erklärungshintergründe haben als eine inhaltliche Überforderung. Wir haben deshalb Frau Bockig noch einmal für eine genauere Beobachtung ausgesucht und ihr ein wenig intensiver über die Schulter gesehen. Es zeigt sich, dass Frau Bockig die Kapitel von vorne nach hinten durchgeht und dass sie dabei einen Grossteil an Inhalten zu bearbeiten hat, die sie bereits kennt. Die ersten 44 Grundlagenkapitel sind ihr aus einem vorangegangenen Kurs vertraut, die einführenden ExcelKapitel sind ihr offensichtlich aufgrund ihrer bisherigen Ausbildung bekannt. Diese Wiederholung der Inhalte führt dazu, dass Frau Bockig sich inhaltlich unterfordert fühlt und ihre Langeweile auf verschiedenste Art 
und Weise äussert. Trotzdem hat sie später in den Prüfungen durchweg Erfolg. Sie besteht die vier ECDL-Start-Prüfungen im ersten Anlauf.

Andererseits ist Frau Bockig methodisch überfordert. Diese Gleichzeitigkeit von inhaltlicher Unterforderung und methodischer Überforderung erscheint mir typisch für E-Learning im Zusammenhang mit gering qualifizierten Teilnehmenden. Die Mischung bei Frau Bockig erweist sich als durchaus explosiv, die inhaltliche Unterforderung in Kombination mit methodischer Überforderung führt zu Aggression, die sich in Lautstärke, Fäkal-Vokabular und exzessiven Nachbarschaftsgesprächen äussert. Die methodische Überforderung zeigt sich in vielen Details. Frau Bockig ist beispielsweise nicht in der Lage, sich mit Hilfe des Inhaltsverzeichnisses relevante Inhalte auszusuchen und die Inhalte zu überspringen, die sie bereits kennt. Das bedeutet auch, dass die Titel des Inhaltsverzeichnisses nicht so klar formuliert sind, dass sie damit etwas anfangen könnte. Das bedeutet weiterhin, dass Frau Bockig nicht auf die Idee kommt, die Titel einmal anzuklicken, um herauszufinden, was sich dahinter verbirgt und dann zu entscheiden, ob sie dort weitermachen möchte oder nicht. Des Weiteren ist Frau Bockig offensichtlich nicht im Stande, neben den für sie langweiligen Inhalten die vielleicht anspruchsvolleren und interessanteren Übungen sowie die Lerntests auszuprobieren. Eine solche suchende, sich orientierende Handlungsweise verlangt eine hohe Konzentration und wäre mit Sicherheit leichter umzusetzen, wenn die Lernenden sie absichtsvoll und bewusst angehen würden.

Hinsichtlich der Projektziele und einer zudem allseits diskutierten Formel lässt sich festhalten, dass das angeblich per E-Learning mittrainierte Selbstlernen (oft synonym und unscharf verwendet werden die Begriffe Selbstlernfähigkeit bzw. Selbstlernkompetenz) sich nicht von selbst einstellt. Die methodische Seite des Lernens muss ebenfalls gelernt werden, wobei wir vorschlagen, hier im Wege der retrospektiven Analyse individuelle und inhaltsbezogene Strategien zu erarbeiten. Dazu wäre die Reflexion auf zurückliegende Lernprozesse hilfreich, mit der die Schritte der Selbstorganisation und der Inhaltsauswahl den Lernenden bewusst werden. So könnte es möglich sein, auch dauerhaft die Selbstlernkompetenz der Betreffenden zu erhöhen und nachhaltiges Weiterlernen per E-Learning anzuregen.

\section{Vorher-Nachher-Befragung}

Kommen wir zu dem Vorwurf, E-Learning sei für eine solche Zielgruppe nicht geeignet. Wir können immerhin anhand der Eingangs- und Ausgangsbefragung einigermassen gut nachvollziehen - und durch die Beobachtung auch auf Plausibilität prüfen - inwiefern Kenntnisse vorher vorgelegen haben und inwiefern sie durch den Kurs mit Hilfe der Lernsoftware erworben wurden.

\begin{tabular}{|lll|}
\hline 5. Können Sie mit Excel eine Tabelle machen? \\
& entry exit \\
& & \\
& 7 & $\mathbf{1 3}$ \\
O Ja, sicher & 4 & $\mathbf{1}$ \\
O Ja, mit etwas Ausprobieren & 0 & 0 \\
O Nein, wohl kaum & 2 & 0 \\
O Nein, kann ich nicht. & & \\
\hline
\end{tabular}

Abbildung 3: Eingangs- und Ausgangsbefragung EXCEL, Frage 5

Anhand der Ergebnistabelle (Abb. 3) ist erkennbar, dass die Beteiligten auf die Frage: «Können Sie eine Tabelle mit Excel machen ${ }^{4}$ ?» anfangs in der Eingangsbefragung höchst unterschiedlich antworten. Sieben Personen sind der Meinung, das könnten sie sicher. Zwei sind der Ansicht, das könnten sie gar nicht. Der Rest verteilt sich. In der Ausgangsbefragung sagen dreizehn von vierzehn Personen: «Ja sicher!» und die verbleibende vierzehnte Person traut sich immerhin mit etwas Ausprobieren zu, eine Tabelle in Excel zu erstellen.
${ }^{4}$ Aufgrund der Sprachschwierigkeiten der Zielgruppe haben wir sämtliche Fragebögen überarbeitet und möglichst einfache deutsche Begriffe verwendet. 
6. Können Sie in Excel Zahlen kopieren?

entry exit

$\begin{array}{lll}\text { O Ja, sicher } & 4 & \mathbf{1 4} \\ \text { O Ja, mit etwas Ausprobieren } & 5 & 0 \\ \text { O Nein, wohl kaum } & 2 & 0 \\ \text { O Nein, kann ich nicht } & 2 & 0\end{array}$

Abbildung 4: Eingangs- und Ausgangsbefragung, Frage 6

Ähnlich ist die Lage bei der Kopieren-Einfügen-Funktion (Abb. 4). Diese Funktion ist für PC-Anfänger zentral, da sie einen entscheidenden Vorteil gegenüber handschriftlicher oder maschinengeschriebenen Tabellen darstellt. Erst mit der PC-Verwendung können Fehler leicht und überall im Dokument gelöscht werden. Zentral ist zudem die Möglichkeit, Teile der Tabelle wieder zu verwenden. Diese Denkweise ist für Anfänger unvertraut, daher haben wir sie exemplarisch aus dem ECDL-Syllabus herausgestellt und operationalisiert. Auch die Fähigkeit, Daten innerhalb einer Excel-Tabelle zu sortieren (Abb. 5), hat die Mehrheit der Lernenden nach eigener Auffassung im Kurs erworben.

Diese Fragen sind durchweg auf den ECDL-Syllabus bezogen und spiegeln wider, was ein Lerner oder eine Lernerin beherrschen muss, um die ECDLPrüfung zu bestehen. Aus dem gesamten Syllabus haben wir je Abschnitt eine exemplarische Frage operationalisiert. Dabei sind wir in den Grundlagen differenzierter vorgegangen als in fortgeschritteneren Bereichen. Die Evaluation zielte nicht darauf, wie viel gelernt wurde, sondern darauf, ob es gelungen ist, besonders die Unerfahrenen und Ungeübten $\mathrm{zu}$ erreichen und ihnen den Anschluss an die Grundlagen der Anwendungssoftware zu ermöglichen.
7. In einer Tabelle stehen die Computer-Preise von verschiedenen Läden. Können Sie die Preise mit Excel so sortieren, dass der günstigste Preis ganz oben steht?

$$
\text { entry exit }
$$

$\begin{array}{lll}\text { O Ja, sicher } & 5 & \mathbf{1 1} \\ \text { O Ja, mit etwas Ausprobieren } & 2 & \mathbf{3} \\ \text { O Nein, wohl kaum } & 3 & 0 \\ \text { O Nein, kann ich nicht } & 3 & 0\end{array}$

Abbildung 5: Eingangs- und Ausgangsbefragung, Frage 7

Darüber hinaus haben wir einige weiterführende Fähigkeiten gesammelt abgefragt (Abb. 6). Auch hier zeigt sich eine deutliche Verbesserung des Bildes. Wir haben jedoch in diesem eher fortgeschrittenen Bereich nicht mehr im Einzelnen nach der Souveränität und Festigkeit der Kenntnisse gefragt. In der Gesamtübersicht zeigt sich nunmehr, dass allen Personen im Kurs die wesentlichen Einstiegsschritte mit Excel gelingen. Es zeigt sich aber auch, dass in den Fortgeschrittenenbereichen einige Differenzen bestehen bleiben. Es gibt hier Bereiche, in denen sich höchstens zehn von vierzehn Personen kompetent fühlen. Das ist der durchaus auch der inhaltlichen Kursorganisation geschuldet, in der die Basiskenntnisse stärker bearbeitet wurden als weiterführende Kenntnisse. Dennoch ist es einigen schnelleren Lernenden gelungen, sich mit der Lernsoftware eigenständig neue Themenbereiche zu erschliessen. 
10. Welche der folgenden Schritte in Excel können Sie? (Angaben gekürzt)

entry exit

$\begin{array}{lrr}\text { O Tabellen formatieren } & 4 & \mathbf{1 4} \\ \text { O Kommastellen definieren } & 2 & \mathbf{1 3} \\ \text { O Schaubilder herstellen } & 2 & \mathbf{1 0} \\ \text { O Grafiken einfügen } & 4 & \mathbf{1 0} \\ \text { O keines davon } & 7 & \mathbf{0}\end{array}$

Abbildung 6: Eingangs- und Ausgangsbefragung, Frage 8

\section{Anregungen und Empfehlungen für E-Learning mit gering qualifizierten Lernenden}

Auf Basis der Erhebung lassen sich einige Empfehlungen aussprechen, die jedoch nicht als empirisch «bewiesen», sondern als offene Problematiken vorgetragen werden. Diese relativ unverbundenen Einzelempfehlungen sind unseres Erachtens als Diskussionsmaterial zu verstehen, mit denen die konkrete Planung eigener Angebote bereichert werden kann. Die bei Benachteiligten mangelnde Selbststeuerungsfähigkeit, die jüngst Schiersmann referiert (2006), ist ein ernst zu nehmendes Lernhindernis und eine pädagogische Herausforderung.

Zunächst ist es zentral das Verhältnis von E-Learning und Präsenz noch einmal durchzudenken. Im Zusammenhang mit E-Learning-Anfängern hat es sich bewährt, mit einem Verhältnis von maximal 50 : 50 (E-Learning : Präsenz) einzusteigen. Das bedeutet nicht, dass dieses Verhältnis so bleiben muss. Es ist aber durchaus notwendig, eine nicht versierte Klientel anfangs intensiv zu begleiten, sie dann zunehmend in die Selbstständigkeit zu entlassen, bzw. zu führen und diese Selbstständigkeit schliesslich von ihr auch zu verlangen. Denkbar und interessant sind Modelle, in denen zunächst Vollzeit im Kurs mit durchgehender tutorielle Begleitung gearbeitet wird, wobei - wie hier - etwa die Hälfte der Zeit mit der Software gearbeitet wird und die andere Hälfte der Zeit der klassischen Unterrichtsmethodik zur Verfügung steht. Im weiteren Verlauf wäre es dann sinnvoll, die Klassenräume zunehmend den Lernenden zu überlassen und die tutiorelle
Betreuung in ihrer Ansprechbarkeit und später auch in ihrer Anwesenheit und für die Lernenden zurückzufahren. Erst in einem dritten Schritt erscheint es sinnvoll, gerade diese Klientel mit der Software in die zeitliche und organisatorische Selbstständigkeit zu schicken. Beim Rückzug in private Räume zum Lernen ist erstens mit schwächerer Computerausstattung zu rechnen und zweitens treten in der Regel häufigere $\mathrm{Ab}$ lenkungsanlässe auf. Eine weitere Empfehlung bezieht sich darauf, die Orientierung innerhalb der Software vor Ort unter Anleitung zu üben. Wir nehmen immer wieder das Phänomen «Lost in Hyperspace» (Verloren im Internet) wahr, dass sich bei ungeübten Nutzern deutlich abbildet.

Weiterhin ist zu erkennen, dass die Lehrkraft ihre Rolle neu definieren muss. Sie moderiert mehr, als dass sie Inhalte übermittelt und präsentiert. Letzteres wird der Software überlassen. Das bedeutet auch, dass die Lehrkraft die Inhalte der Software sehr gut kennen muss, um auf Fragen der Teilnehmenden eingehen zu können, selbst wenn 15 Personen aus den 15 verschiedenen Kapiteln, in denen sie sich bewegen, ihre Fragen stellen. Eingestreute Präsenzphasen dienten hier sehr erfolgreich als Pegel, anhand dessen gemessen wurde, wer wie gut mit seinen E-Learning-Aufgaben zurecht kommt und wie weit Einzelne innerhalb der Gruppe bisher gelangt sind.

Wichtig erscheint bei der Einführung von E-Learning für neue Zielgruppen oder in neuen Bildungsinstitutionen, dass die Entscheidung für oder gegen E-Learning nicht schon nach dem ersten Versuch fällt, sondern dass dem E-Learning ein zweiter oder auch dritter Durchlauf gewährt wird. Wir nehmen aus der Evaluation wahr, dass die anfängliche Widerständigkeit gegen E-Learning sich sowohl bei den Trainern als auch bei Teilnehmern im zweiten und dritten Durchgang verflüchtigt. Das hat nicht zuletzt damit zu tun, dass die Abläufe und die Navigation innerhalb der Software nach und nach vertrauter werden.

Nicht zu unterschätzen ist nach wie vor das Frustrationspotenzial der Einzelarbeit. Selbst im Gruppenraum, in der Nachbarschaft mit der Bankreihe wird immer wieder erkennbar, dass die Sorge, allein am Problem zu scheitern die Teilnehmenden erheblich stärker frustriert, als wenn im synchronen Unterricht alle an derselben Übung arbeiten und sich kurz rechts und links vergewissern können, dass die anderen an ähnlichen Schwierigkeiten scheitern, oder dass ähnliche Missverständnisse aufgetreten sind. 
Komplementär zur Moderationsrolle der Lehrenden findet sich ein Verantwortungstransfer auf Lernende. Auch dieser muss begleitet und betreut werden. Er darf jedoch nicht durch einen überbetreuenden «Pampers Approach» wieder zurück genommen werden.

Schwierig ist und bleibt trotz der Chancen der Binnendifferenzierung, die durch E-Learning gegeben sind, die notwendige Gruppensynchronisierung in Phasen der gemeinsamen Arbeit. Wenn sich die Gruppe in vielen verschiedenen Kapiteln zugleich aufhält, wird es jeder Lehrkraft schwer fallen $\mathrm{zu}$ entscheiden, welcher Inhalt allen Beteiligten fehlt, um diesen in einer gemeinsamen Gruppenarbeit synchron zu bearbeiten oder vorzutragen. Insgesamt verzeichnen wir ein widerständiges Einlassen auf E-Learning, das einerseits Reibungsverluste beinhaltet, andererseits Annäherungen sowohl der Lehrenden als auch der Lernenden erkennen lässt.

Nimmt man das gesamte Projekt noch einmal in den Blick, dann sind die Ziele in unterschiedlichem Grade erfüllt. Telelernen fand über die erhobenen 45 Personen hinaus bei mehreren hundert Personen statt. Gering qualifiziert waren die Teilnehmenden durchweg, dies liess sich durch die Eingangserhebung und die Auswahl der Teilnehmenden klären. Zum Ziel <erhöhte Selbstlernkompetenzen〉 bleibt jedoch zu sagen, dass Frau Bockig, Frau Unsichtbar und Herr Selfmade noch einiges mehr an expliziter Kenntnis ihrer selbstbezüglichen pädagogischen Handlungen benötigen. Sie haben alle drei zwar Fortschritte gemacht, jedoch ist ihnen das kaum Weise bewusst. Noch weniger sind sie in der Lage, verbal auszudrücken, was sie gelernt haben und wie sie es gelernt haben. Dies sollte durch zunehmende Reflexion auf die gerade vergangenen Lernschritte stärker eingeführt werden.

\section{Literatur}

Barz, Heiner; Tippelt, Rudolf (Hrsg.): Adressaten- und Milieuforschung zu Weiterbildungsverhalten und -interessen. Bielefeld 2004.

Baumert, Jürgen; Stanat, Petra; Demmrich, Anke: PISA 2000. Untersuchungsgegenstand, theoretische Grundlagen und Durchführung der Studie. Aus: PISA 2000: Basiskompetenzen von Schülerinnen und Schülern im internationalen Vergleich. 2001. S. 15-68.

Bourdieu, Pierre: Die feinen Unterschiede. Suhrkamp Verlag Frankfurt 1987.
Ehmann, Christoph: Bildungsfinanzierung und soziale Gerechtigkeit. Vom Kindergarten bis zur Weiterbildung. 2., überarbeitete Auflage Bielefeld 2003

Expertenkommission Finanzierung Lebenslangen Lernens: Der Weg in die Zukunft. Schlussbericht. Bielefeld 2004. (= Schriftenreihe der Expertenkommission Finanzierung Lebenslangen Lernens. 6)

Faulstich, Peter: Arbeitsorientierte Erwachsenenbildung. Frankfurt a. M., Berlin, München 1981.

Feller, Gisela: wbmonitor: Ein Panel zum heterogenen Markt beruflicher Weiterbildung und Sprachrohr für Anbieter. Aus: Bos, Wilfried; Lankes, Eva-Maria; Plassmeier, Nike u. a. (Hrsg.): Heterogenität. Eine Herausforderung an die empirische Bildungsforschung. Münster, New York, München u. a. 2004. S. 117-126.

Grotlüschen, Anke; Brauchle, Barbara: Bildung als Brücke für Benachteiligte. Hamburger Ansätze zur Überwindung der Digitalen Spaltung. Evaluation des Projekts ICC - Bridge to the Market. Münster (LIT) 2004.

Holzkamp, Klaus (1993): Lernen. Subjektwissenschaftliche Grundlegung. Campus Verlag Frankfurt, New York

Holzkamp, Klaus (1997): Lernen. Subjektwissenschaftliche Grundlegung. Einführung in das Hauptanliegen des Buches (1996), in: Schriften I. Normierung, Ausgrenzung, Widerstand. Argument Verlag Hamburg.

Kuwan, Helmut: Weiterbildungseinstellungen, Weiterbildungsbarrieren und Weilterbildungsteilnahme in Deutschland. Gruppenspezifisch differenzierende Analysen auf Basis einer telefonischen Repräsentativbefragung. Aus: Bos, Wilfried; Lankes, Eva-Maria; Plassmeier, Nike u. a. (Hrsg.): Heterogenität. Eine Herausforderung an die empirische Bildungsforschung. Münster, New York, München u. a. 2004. S. 173194.

Kuwan, Helmut; Thebis, Frauke: Berichtssystem Weiterbildung IX. Ergebnisse der Repräsentativbefragung zur Weiterbildungssituation in Deutschland. München 2004.

Lehmann, Rainer H.; Peek, Rainer; Gänsfuss, Rüdiger: Aspekte der Lernausgangslage und der Lernentwicklung. Bericht über die Erhebung im September 1996 (LAU 5).

www.hamburger-bildungsserver.de/schulentwicklung/lau/lau5, Funddatum 01.11.2004 1997. 
Michel, Lutz P.; Pelka, Bastian: Marktumfeld und Marktentwicklung. Aus: Dohmen, Dieter; Michel, Lutz P. (Hrsg.): Marktpotenziale und Geschäftsmodelle für eLearning-Angebote deutscher Hochschulen. Bielefeld (wbv) 2003. S. 93-144.

Pinscher, Rainer; Wagner, Gert G.; Haisken-DeNew, John: DIW-Wochenbericht 41/00. Computer und Internetbenutzung hängen stark von Einkommen und Bildung ab - Geschlechterspezifische Nutzungsunterschiede in der Freizeit besonders ausgeprägt. In: Internet http://www.diw.de/deutsch/publikationen/ wochenberichte/docs/00-412.html 16.05.2002, Jg. 2000, S. 1-8.

Schiersmann, Christiane: Profile lebenslangen Lernens. Bielefeld, wbv 2006.

Schröder, Helmut; Schiel, Stefan; Aust, Folkert: Nichtteilnahme an beruflicher Weiterbildung. Motive, Beweggründe, Hindernisse. Bielefeld 2004. (= Schriftenreihe der Expertenkommission Finanzierung Lebenslangen Lernens. 5)

Tippelt, Rudolf; Reich, Jutta: Weiterbildungsinteressen und Weiterbildungsverhalten: Bildungsorientierungen und Weiterbildungsbarrieren. Aus: Bos, Wilfried; Lankes, Eva-Maria; Plassmeier, Nike u. a. (Hrsg.): Heterogenität. Eine Herausforderung an die empirische Bildungsforschung. Münster, New York, München u. a. 2004. S. 281-300.

Tippelt, Rudolf; Weiland, Meike; Panyr, Sylva u. a.: Weiterbildung, Lebensstil und soziale Lage in einer Metropole. Studie zu Weiterbildungsverhalten und -interessen der Münchner Bevölkerung. Bielefeld (wbv) 2003.

TNS Emnid, Initiative D21: 53 Prozent der Deutschen sind online! In: www.nonliner-atlas.de, 01.11.2004,

Vester, Michael: Die Illusion der Bildungsexpansion. Bildungsöffnungen und soziale Segregation in der Bundesrepublik Deutschland. Aus: Krais, Beate; Engler, Steffani (Hrsg.): Das kulturelle Kapital und die Macht der Klassenstrukturen. Sozialstrukturelle Verschiebungen und Wandlungsprozesse des Habitus. Weinheim und München 2004. 